\title{
A Standard Cytogenetic Photomap for the Mosquito Anopheles stephensi (Diptera: Culicidae): Application for Physical Mapping
}

\author{
MARIA V. SHARAKHOVA, AI XIA, SARAH I. MCALISTER, AND IGOR V. SHARAKHOV ${ }^{1}$
}

Department of Entomology, Virginia Tech, Blacksburg, VA 24061

\section{J. Med. Entomol. 43(5): 861-866 (2006)}

\begin{abstract}
To facilitate physical genome mapping, we have developed a new cytogenetic photomap for Anopheles stephensi (Liston) (Diptera: Culicidae), an important malaria vector in Asia. The high-resolution images of the ovarian polytene chromosomes have been straightened and divided by numbered divisions and lettered subdivisions. The exact chromosomal locations of eight DNA probes have been determined by fluorescent in situ hybridization. Using the DNA sequences, we have established correspondence between chromosomal arms among An. stephensi, Anopheles gambiae (Patton), and Anopheles funestus (Giles). The results support previous cytogenetic observations of arm translocations taking place during diversification of the species. To make the cytogenetic map useful for population genetics studies, we have indicated the chromosomal positions for the breakpoints of 19 polymorphic inversions.
\end{abstract}

KEY WORDS Anopheles stephensi, cytogenetic photomap, polytene chromosomes, fluorescent in situ hybridization

Anopheles stephensi (Liston) (Diptera: Culicidae) is an important malaria vector in the Indo-Pakistan subcontinent and the Middle East. As for the major malaria vectors in Africa, Anopheles gambiae (Patton) and Anopheles funestus (Giles), An. stephensi is a member of the subgenus Cellia. The three species belong to the different series within Cellia: Pyretophorus ( $A n$. gambiae), Myzomyia (An. funestus), and Neocellia (An. stephensi) (Coluzzi et al. 1970, Green and Hunt 1980).

Among anopheline species, An. stephensi is the most convenient choice for experimental genetic studies. Unlike other mosquitoes, it can be readily bred in the laboratory and efficiently infected both with human and rodent Plasmodium species. Use of An. stephensi as a model system has allowed significant progress in studying mosquito-parasite interactions (Han et al. 2000, Abraham et al. 2004, Lim et al. 2005). Also, the biggest success with stable genetic germline transformation and heritable RNA interference has been achieved for this Anopheles mosquito (Catteruccia et al. 2000, Brown et al. 2003). However, the lack of sufficient genomic information for An. stephensi prevents researchers from taking full advantage of this species as a model system. For example, a physical genome map could serve as a useful tool for the localization and isolation of genes of interest by positional or map-based cloning.

\footnotetext{
${ }^{1}$ Corresponding author, e-mail: igor@vt.edu.
}

There have been few successful attempts to map genes to the An. stephensi chromosomes by in situ hybridization (Garvey and Malcolm 2000, Andreasen and ffrench-Constant 2002). In some cases, localization to a specific chromosome was not possible because of limited information available on chromosome banding patterns in this species (Luckhart and Rosenberg 1999). There has been a need for a standard cytogenetic photomap that can be used for physical mapping of the An. stephensi genome.

An. stephensi has a typical karyotype for Anopheles, $2 n=6$ and five well-developed arms of polytene chromosomes in its salivary glands and ovarian nurse cells. The presence of high-quality polytene chromosomes in both tissues makes this species a superior model for cytogenetic research. Several cytogenetic maps have been developed for An. stephensi for use in cytotaxomony and population genetics studies of inversion polymorphism. The first drawn and photomaps of the chromosomes from the salivary glands were developed by Sharma et al. (1969). A year later, the first photomap of the ovarian nurse cell chromosomes was published by Coluzzi et al. (1970). The authors indicated that the banding pattern was found to be more constant and clear in the ovarian chromosomes than in the salivary gland chromosomes. The former also were found easier to prepare and should be considered a more favorable material for cytogenetic studies. The photomap by Coluzzi et al. (1970) was divided into 46 numbered divisions but not subdivided into subdivisions. In addition, the chromo- 
some images were not straightened. The first standard drawn map of ovarian nurse cell chromosomes of An. stephensi was developed by Mahmood and Sakai (1985). The numbered divisions were retained as proposed by Coluzzi et al. (1970). The authors divided the map further into 151 lettered subdivisions and described the landmarks for all chromosomes in detail. They also located breakpoints for 19 paracentric inversions that are present in natural and laboratory populations of An. stephensi in India (Coluzzi et al. 1970, 1973; Mahmood and Sakai 1984). Studies of inversion polymorphisms assist in our understanding of the genetic structure of natural populations. For example, comparison of urban and rural populations of An. stephensi revealed differences in the types and frequencies of paracentric inversions (Mahmood and Sakai 1984). Subbarao (1996) has published a photomap of An. stephensi with the localization of the breakpoints of 27 polymorphic inversions. However, this map was not divided into numbered and lettered regions.

Thus, all the cytogenetic maps that have been available for the polytene chromosomes from the ovarian nurse cells of An. stephensi are either drawn or without divisions and subdivisions. Although these maps have been used for identification of polymorphic inversions, they are not useful for physical mapping. Straightened high-resolution images of polytene chromosomes with numbered divisions and lettered subdivisions are needed for the efficient interpretation of in situ hybridization results. For this reason, we developed a new cytogenetic photomap and used it for the mapping of several genes to the An. stephensi chromosomes.

\section{Materials and Methods}

Mosquito Strain and Tissue Preservation. We used the Indian wild-type strain of An. stephensi, which is a standard laboratory strain used in other studies (Luckhart and Rosenberg 1999, Lim et al. 2005). Ovaries were pulled out from half-gravid females and preserved in Carnoy's fixative solution ( 3 ethanol:1 glacial acetic acid by volume). Ovaries were fixed for $24 \mathrm{~h}$ at room temperature and stored at $\left[\right.$ minus $20^{\circ} \mathrm{C}$ until use.

Chromosome Preparation and Analysis. Ovaries from half-gravid females prefixed in Carnoy's fixative solution were dissected in $50 \%$ propionic acid. A coverslide was placed on the follicles and pressed to squash the cells. The banding pattern of polytene chromosomes was examined using a Leica phase-contrast microscope $(1,000 \times)$ (Leica, Wetzlar, Germany). Slides with good chromosomal preparations were dipped in liquid nitrogen, and then coverslips were removed and slides were dehydrated in 50, 70, 95, and $100 \%$ ethanol. To take images, we applied a drop of $100 \%$ ethanol to a dry chromosomal preparation and covered it with a coverslip. Images were taken with the Olympus Q-color5 digital cooled five megapixel camera (Olympus America Inc., Melville, NY). We chose 12 chromosome preparations from a total of 160 for the cytogenetic map construction. Images of all five chromosomal arms have been straightened using Adobe Photoshop software (Adobe Software, Mountain View, CA).

Probe Preparation. Primers were designed based on the An. stephensi cDNA sequences available from the GenBank (http://ncbi.nih.gov/Genbank/). For the design, we used Primer three software (http:/ / frodo. wi.mit.edu/cgi-bin/primer3/primer3 www.cgi). The genomic DNA of single mosquitoes was extracted using the Wizard SV Genomic Purification System (Promega, Madison, WI). Polymerase chain reaction (PCR) products were gel purified using the GeneClean kit (Qbiogene Inc., Carlsbad, CA). Genomic inserts from the An. funestus SMART cDNA library (Sharakhov et al. 2002) were PCR amplified using T3/T7 or Amplimer primers. The PCR conditions with the Amplimer primers were $95^{\circ} \mathrm{C}$ for $5 \mathrm{~min} ; 25$ cycles of $94^{\circ} \mathrm{C}$ for $30 \mathrm{~s}, 70^{\circ} \mathrm{C}$ for $2 \mathrm{~min} ; 68^{\circ} \mathrm{C}$ for $3 \mathrm{~min}$. The PCR conditions with the $\mathrm{T} 3 / \mathrm{T} 7$ primers were $95^{\circ} \mathrm{C}$ for $5 \mathrm{~min} ; 25$ cycles of $94^{\circ} \mathrm{C}$ for $30 \mathrm{~s}, 50^{\circ} \mathrm{C}$ for $30 \mathrm{~min} ; 72^{\circ} \mathrm{C}$ for $30 \mathrm{~s} ; 72^{\circ} \mathrm{C}$ for $5 \mathrm{~min}$. The DNA was labeled with Cy5-AP3-dUTP (GE Healthcare, Little Chalfont, Buckinghamshire, United Kingdom) using Random Primers DNA Labeling System (Invitrogen, Carlsbad, CA)

Fluorescent In Situ Hybridization. Fixed ovaries were gently pressed with a coverslip in $50 \%$ propionic acid and dipped in liquid nitrogen. Then, the coverslips were removed, and the slides were dehydrated in 50,70, 95, and $100 \%$ ethanol. DNA probes were hybridized to the chromosomes at $39^{\circ} \mathrm{C}$ overnight in hybridization solution (Invitrogen). Then, the chromosomes were washed in $0.2 \times$ saline sodium citrate $(0.03 \mathrm{M}$ sodium chloride and $0.003 \mathrm{M}$ sodium citrate), counterstained with YOYO-1 (Invitrogen), and mounted in 1,4-diazabicyclo[2.2.2] octane (SigmaAldrich, St. Louis, MO). Fluorescent signals were detected and recorded using a Zeiss LSM 510 laser scanning microscope (Carl Zeiss MicroImaging, Inc. Thornwood, NY).

\section{Results}

Photomap of Chromosomes. We prepared a photomap of the straightened An. stephensi polytene chromosomes with numbered divisions and lettered subdivisions (Fig. 1). The borders and nomenclature for divisions and subdivisions as well as the notations for the polymorphic inversions have been adopted from a drawn map (Mahmood and Sakai 1985). Below is the description of the landmarks useful for arm identification.

The $\mathrm{X}$ chromosome is the shortest in the complement. It can be easily recognized by its flared, lightly stained telomeric end (region 1A) and two wide heterochromatic areas on the centromeric end (regions $6 \mathrm{~A}$ and $6 \mathrm{~B}$ ). Usually, the $\mathrm{X}$ chromosome is not connected to the other arms in the squashes of An. stephensi ovarian nurse cells.

The telomeric region $7 \mathrm{~A}$ of the longest arm $2 \mathrm{R}$ has three distinct, narrow bands at the end. There are 


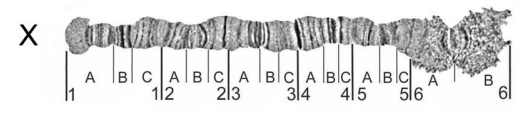

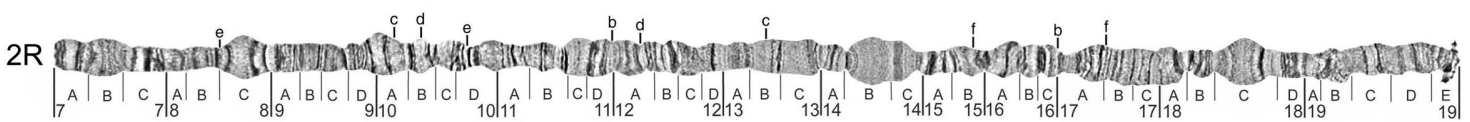

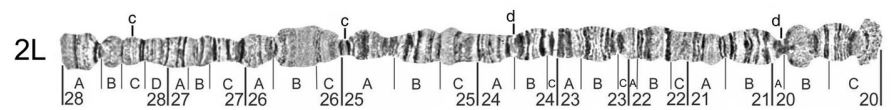

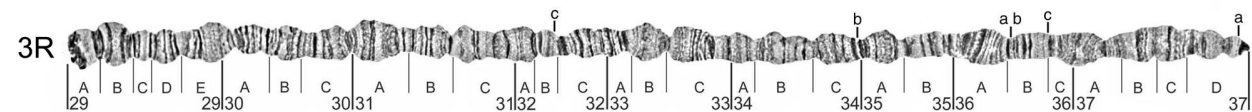

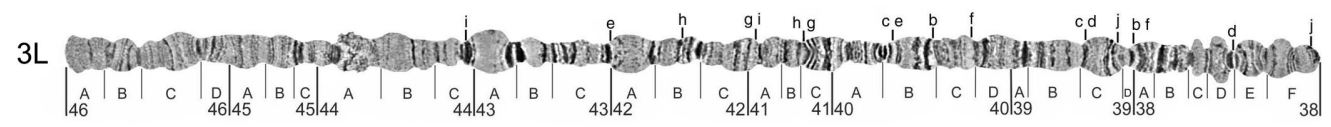

Fig. 1. Standard photomap of the polytene chromosomes from the An. stephensi ovarian nurse cells. Positions of breakpoints for polymorphic inversion are shown above the chromosomes.

several large puffs along the chromosome. The region $8 \mathrm{C}$ is marked by a light puff surrounded by dark bands at both ends. The very large round puff in region 14B is a very prominent landmark for the $2 \mathrm{Rb}$ paracentric inversion. The Indian wild-type strain is polymorphic for this inversion. The centromeric end (19D) is usually granulated and flared. The pericentromeric area can be recognized by a diffuse region 19B, five thin bands in 18D, and a puff in 18C.

The telomeric end of the $2 \mathrm{~L}$ arm contains two dark, narrow bands in the proximal part of region 28A. This chromosome has a very narrow zone in region $20 \mathrm{~A}$ and a large diffuse area in region 20B. The centromeric end of $2 \mathrm{~L}$ is flared, light, and granulated. The right and left

Table 1. DNA probes used for in situ hybridization with the An. stephensi polytene chromosomes

\begin{tabular}{|c|c|c|c|c|c|c|}
\hline & Clone name & Accession no. & Description & $\begin{array}{l}\text { Location in } \\
\text { An. stephensi }\end{array}$ & $\begin{array}{l}\text { Location in } \\
\text { An. gambiae }\end{array}$ & $\begin{array}{l}\text { Location in } \\
\text { An. funestus }\end{array}$ \\
\hline 1 & As_gSG6 & AY226456 & $\begin{array}{l}\text { gSG6 salivary gland protein } \\
\text { precursor }\end{array}$ & $\mathrm{X}, 1 \mathrm{~A}$ & $\mathrm{X}, 4 \mathrm{~B}(4.1 \mathrm{e}-09)$ & ND \\
\hline 2 & 26_G11 & BU038979 & Ribosomal protein L10 & $\mathrm{X}, 4 \mathrm{~B}$ & $\begin{array}{l}\mathrm{X}, 5 \mathrm{C}^{*} \\
(2.4 \mathrm{e}-184) \\
2 \mathrm{R}, 12 \mathrm{~B} \\
(5.7 \mathrm{e}-156)\end{array}$ & $\begin{array}{l}\mathrm{X}, 1 \mathrm{~B}^{*} \\
2 \mathrm{~L}, 28 \mathrm{C}\end{array}$ \\
\hline 3 & 11_D07 & BU038904 & C-4 methyl sterol oxidase & $2 \mathrm{R}, 15 \mathrm{~A}$ & $\begin{array}{l}2 \mathrm{R}, 14 \mathrm{D}^{*} \\
(1.5 \mathrm{e}-190) \\
\mathrm{X}, 5 \mathrm{D} \\
(1.1 \mathrm{e}-07)\end{array}$ & $2 \mathrm{R}, 13 \mathrm{~A}$ \\
\hline 4 & 03_G10 & BU038875 & Hexamerin A & $2 \mathrm{~L}, 22 \mathrm{C}$ & $\begin{array}{l}3 \mathrm{~L}, 39 \mathrm{~B} \\
(1.5 \mathrm{e}-126)\end{array}$ & $3 \mathrm{~L}, 41 \mathrm{~A}$ \\
\hline 5 & Ski & AY578814 & $\begin{array}{l}\text { Sloan-Kettering Institute } \\
\text { proto-oncogene product }\end{array}$ & $3 \mathrm{R}, 29 \mathrm{~B}$ & 3R:29A (1.5e-207) & ND \\
\hline 6 & 19_F10 & BU038947 & Isocitrate dehydrogenase & $3 \mathrm{R}, 29 \mathrm{~B}$ & 3R, 29A (6.1e-172) & $2 \mathrm{~L}, 27 \mathrm{D}$ \\
\hline 7 & PPO1 & AY559300 & Prophenoloxidase 1 & $3 \mathrm{~L}, 40 \mathrm{D}$ & $\begin{array}{l}2 \mathrm{~L}, 24 \mathrm{~A}^{*}(1.4 \mathrm{e}-118) \\
2 \mathrm{~L}, 20 \mathrm{D}(3.4 \mathrm{e}-13) \\
2 \mathrm{R}, 13 \mathrm{~B} \\
(2.1 \mathrm{e}-11)\end{array}$ & ND \\
\hline 8 & 26_A01 & BU038977 & $\begin{array}{l}\text { SH3 domain binding glutamic } \\
\text { acid-rich protein }\end{array}$ & $3 \mathrm{~L}, 46 \mathrm{C}$ & $\begin{array}{l}2 \mathrm{~L}, 27 \mathrm{D} \\
(1.0 \mathrm{e}-110)\end{array}$ & $3 \mathrm{R}, 30 \mathrm{~A}$ \\
\hline
\end{tabular}

ND, not determined; SH3, Src homology 3.

The e-values are indicated in parentheses below the locations. Asterisk indicates major BLASTN hit (in An. gambiae) or hybridization signal (in An. funestus). 


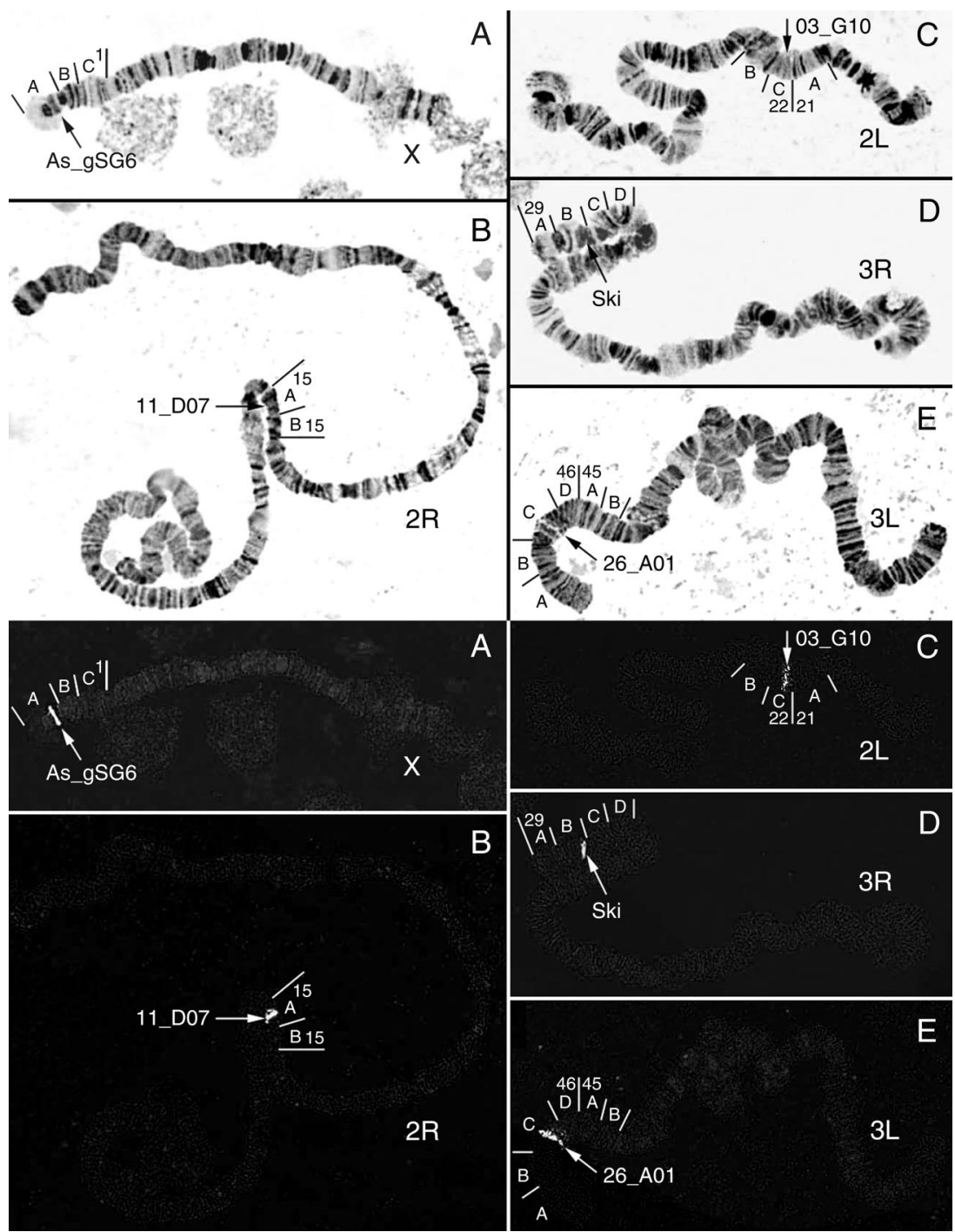

Fig. 2. In situ hybridization of Cy5 fluorescently labeled DNA probes performed on the chromosomes of An. stephensi. Top, banding pattern of the chromosomes. Bottom, fluorescence due to hybridization. Probes and sites of hybridization are as follows: (A) As gSG6 mapped to 1A, chromosome X; (B) 11 D07 mapped to 15A, arm 2R; (C) 03 G10 mapped to $22 \mathrm{C}$, $\operatorname{arm} 2 \mathrm{~L}$; (D) Ski mapped to 29B, arm 3R; and 26_mapped to 46C, arm 3L. Chromosomes counterstained with the fluorophore YOYO-1.

arms of chromosome two are often found lying separately on the slide.

The 3R arm can be recognized by a dark, granulated, flared telomeric end and a round puff with two dark bands in region 29B. This chromosomal arm does not have any other large puffs. The centromeric end of the $3 \mathrm{R}$ arm has a very dark and narrow band in the most proximal part of region 37D.

The $3 \mathrm{~L}$ arm has a very light telomeric end and two large puffs in divisions $42 \mathrm{~A}$ and $43 \mathrm{~A}$. The puff in region $43 \mathrm{~A}$ is framed by large, dark bands. There is a wide granulated area in division $44 \mathrm{~A}$. The pericentromeric region of the $3 \mathrm{~L}$ arm contains five small puffs in $38 \mathrm{C}-\mathrm{F}$ and a narrow proximal end. The left and the right arms are usually connected on the chromosomal preparations.

Physical Mapping. Table 1 shows a list of eight DNA probes we used for the physical mapping. Each of these probes has been mapped to a single location on one of the five arms of the An. stephensi polytene chromosome complement. The exact location of each signal on the chromosomes was determined according to the An. stephensi cytogenetic map (Fig. 2). No variation in signal localization was ever detected among all the nuclei examined for a given probe. Three probes were PCR-amplified fragments of An. stephensi genomic DNA. We have used primer designs based on the An. stephensi cDNA sequences available from the 
National Center for Biotechnology Information GeneBank (http://ncbi.nih.gov/Genbank/). The other five probes (26 G11, 11 D07, 03 G10, 19 F10, and 26 A01) were inserts from the An. funestus SMART cDNA library with known locations on the An. funestus chromosomes (Sharakhov et al. 2002). Locations of the sequences in the An. gambiae genome were determined by BLASTN against the Ensembl database (http://www.ensembl.org/Multi/blastview?species= Anopheles gambiae). Three of these genes (26 G11, 11_D07, and PPO1) are part of larger gene families with two or more chromosomal locations in An. gambiae (Table 1). Members of the corresponding protein families are listed in the AnoXcel database (Ribeiro et al. 2004). We have been able to identify the major BLASTN hits based on the score and e-value.

\section{Discussion}

To facilitate physical mapping of the An. stephensi genome, we have developed a standard cytogenetic photomap. The photomap provides more details on banding patterns than the drawn maps. Moreover, in contrast to previous photomaps, the images of polytene chromosomes are straightened and marked with numbered divisions and lettered subdivisions.

BLASTN of the An. funestus cDNA sequences against the Ensembl database (http://www.ensembl. org/Multi/blastview?species=Anopheles_gambiae) has determined the presence of homologous sequences in the An. gambiae genome. Using the An. funestus DNA probes, we have shown the feasibility of interspecific in situ hybridization with the An. stephensi chromosomes. We have demonstrated the usefulness of the map for locating the fluorescent signals to the chromosomal subdivisions (Fig. 2). Our data on in situ hybridization of eight probes is in agreement with the conclusion on arm correspondence between An. stephensi and An. gambiae derived from the previous mapping results (Garvey and Malcolm 2000, Andreasen and ffrench-Constant 2002). Together with the genome mapping data for An. funestus (Sharakhov et al. 2002) these results support the cytological observations about whole arm translocations in subgenus Cellia (Coluzzi et al. 1970, Green and Hunt 1980). Accordingly, chromosomes X and 2R are homologous across all three species. The $2 \mathrm{~L}$ arm of An. gambiae corresponds to the 3R of An. funestus and the $3 \mathrm{~L}$ of An. stephensi. The $2 \mathrm{~L}$ arm of An. stephensi corresponds to the $3 \mathrm{~L}$ arms of $A n$. funestus and An. gambiae. The 2L arm of An. funestus corresponds to the 3R arms of An. gambiae and An. stephensi. Phylogenetic relations for these three species have been estimated from ribosomal DNA (rDNA) (18S and 28S) sequence data (Marshall et al. 2004). Accordingly, An. funestus and An. gambiae are more distant species from each other than from An. stephensi.

To make this map applicable for population genetics studies, we indicated chromosomal positions for the breakpoints of the 19 paracentric inversions described for An. stephensi (Coluzzi et al. 1970, 1973; Mahmood and Sakai 1984). Interestingly, polymorphic inversions are not distributed evenly across the An. stephensi genome. More than two-thirds of them are distributed among two arms. Five inversions are on $2 \mathrm{R}$ and nine on 3L. Nonrandom distribution of polymorphic inversions has been described for other species. For example, of eight polymorphic inversions described in An. gambiae s.s., seven occur on chromosome 2R (Coluzzi et al. 1979). Eleven of 17 polymorphic inversions found in An. funestus involve $2 \mathrm{R}$ (Sharakhov et al. 2004). Physical mapping of the An. stephensi genome is in progress in our lab. Comparison of the linear gene orders among species should yield valuable insights about the pattern of fixed inversion in mosquito evolution.

\section{Acknowledgments}

We thank Nora Besansky for providing the genomic inserts from the An. funestus SMART cDNA library. We thank Zhijian Jake Tu and Thomas R. Saunders for supplying the An. stephensi females. The work was supported by the Agricultural Experimental Station at Virginia Tech (Blacksburg, VA).

\section{References Cited}

Abraham, E. G., S. Islam, P. Srinivasan, A. K. Ghosh, J. G. Valenzuela, J.M.C. Ribeiro, F. C. Kafatos, G. Dimopoulos, and M. Jacobs-Lorena. 2004. Analysis of the Plasmodium and Anopheles transcriptional repertoire during ookinete development and midgut invasion. J. Biol. Chem. 279: 5573-5580.

Andreasen, M. H., and R. H. ffrench-Constant. 2002. In situ hybridization to the $\mathrm{Rdl}$ locus on polytene chromosome 3L of Anopheles stephensi. Med. Vet. Entomol. 16: 452455.

Brown, A. E., L. Bugeon, A. Crisanti, and F. Catteruccia. 2003. Stable and heritable gene silencing in the malaria vector Anopheles stephensi. Nucleic Acids Res. 31: e85.

Catteruccia, F., T. Nolan, T. G. Loukeris, C. Blass, C. Savakis, F. C. Kafatos, and A. Crisanti. 2000. Stable germline transformation of the malaria mosquito Anopheles stephensi. Nature (Lond.) 405: 959-962.

Coluzzi, M., G. Cancrini, and M. Di Deco. 1970. The polytene chromosomes of Anopheles superpictus and relationships with Anopheles stephensi. Parassitologia 12: 101112.

Coluzzi,M., M. Di Deco, and G. Cancrini. 1973. Chromosomal inversions in Anopheles stephensi. Parassitologia 15: 129-136.

Coluzzi, M., A. Sabatini, V. Petrarca, and M. A. Di Deco. 1979. Chromosomal differentiation and adaptation to human environments in the Anopheles gambiae complex. Trans. R. Soc. Trop. Med. Hyg. 73: 483-4 97.

Garvey, C. F., and C. A. Malcolm. 2000. Anopheles stephensi Dox-A2 shares common ancestry with genes from distant groups of eukaryotes encoding a $26 \mathrm{~S}$ proteasome subunit and is in a conserved gene cluster. J. Mol. Evol. 50: 497509.

Green, C. A., and R. H. Hunt. 1980. Interpretation of the variation in ovarian polytene chromosomes of Anopheles funestus Giles, A. parensis Gillies, and A. aruni?. Genetica 51: 187-195.

Han, Y. S., J. Thompson, F. C. Kafatos, and C. Barillas-Mury. 2000. Molecular interactions between Anopheles stephensi midgut cells and Plasmodium berghei: the time bomb theory of ookinete invasion of mosquitoes. EMBO J. 19: $6030-6040$. 
Lim, J., D. C. Gowda, G. Krishnegowda, and S. Luckhart. 2005. Induction of nitric oxide synthase in Anopheles stephensi by Plasmodium falciparum: mechanism of signaling and the role of parasite glycosylphosphatidylinositols. Infect. Immun. 73: 2778-2789.

Luckhart, S., and R. Rosenberg. 1999. Gene structure and polymorphism of an invertebrate nitric oxide synthase gene. Gene 232: 25-34.

Mahmood, F., and R. K. Sakai. 1984. Inversion polymorphisms in natural populations of Anopheles stephensi. Can. J. Genet. Cytol. 26: 538-546.

Mahmood, F., and R. K. Sakai. 1985. An ovarian chromosome map of Anopheles stephensi. Cytobios 43: 79-86.

Marshall, J. C., J. R. Powell, and A. Caccone. 2005. Short report: phylogenetic relationships of the anthropophilic Plasmodium falciparum malaria vectors in Africa. Am. J. Trop. Med. Hyg. 73: 749-752.

Ribeiro, J.M.C., P. Topalis, and C. Louis. 2004. AnoXcel: an Anopheles gambiae protein database. Insect Mol. Biol. 13: 449-457.
Sharakhov, I. V., A. C. Serazin, O. G. Grushko, A. Dana N. F. Lobo, M. E. Hillenmeyer, R. Westerman, J. Romero-Severson, C. Costantini, N. Sagnon, et al. 2002. Inversions and gene order shuffling in Anopheles gambiae and A. funestus. Science (Wash., DC) 298: 182-185.

Sharakhov, I., O. Braginets, O. Grushko, A. Cohuet, W. M. Guelbeogo, D. Boccolini, M. Weill, C. Costantini, N. Sagnon, D. Fontenille, et al. 2004. A microsatellite physical map of the African human malaria vector Anopheles funestus. J. Hered. 95: 29-34.

Sharma, G. P., R. Parshad, S. L. Narang, and J. B. Kitzmiller. 1969. The salivary chromosomes of Anopheles stephensi. J. Med. Entomol. 6: 68-71.

Subbarao, S. K. 1996. Genetics of malaria vectors. Proc. Natl. Acad. Sci. India 66: 51-76.

Received 8 February 2006; accepted 21 June 2006. 